\title{
Electron microscopy study of chemically deposited Ni-P films
}

\author{
S V S TYAGI, V K TANDON and S RAY* \\ Department of Physics, "Department of Metallurgy, University of Roorkee, Roorkee 247667 , \\ India

\begin{abstract}
The structure of electroless thin films of Ni-P has been studied. The microstructure and the selected area diffraction pattern of the samples reveal that certain samples transform to crystalline $\mathrm{N} i$ with $\mathrm{P}$ in solid solution by nucleation and growth, whereas others transform to crystalline state by growth alone. The former set of thin films having a P-content of $19-21$ at. $\%$ is characterized as amorphous. Films with a P-content of $13-15$ at. $\%$ fall in the latter category and are characterized as microcrystalline. Those with a P-content of $16-18$ at \% contain both amorphous and microcrystalline regions.
\end{abstract}

Keywords. Amorphous, microcrystalline; electroless; nickel-phosphorous; electron microscopy.

\section{Introduction}

Electroless deposition of $\mathrm{Ni}$ with different metalloids like $\mathrm{P}, \mathrm{B}$ etc has produced both crystalline and amorphous thin films depending upon the composition of the bath (Pai and Marton 1972; Gorbunova et al 1973; Hedgecock et al 1975; Yamasaki et al 1981; Cortiju and Schlesinger 1983). These metalloids have very restricted solid solubilities in Ni (Maeda 1970). When the amuunt of metalloid exceeds the limit of solid solubility, the film at equilibrium has a suitable precipitate containing the excess metalloid in the matrix of $\mathrm{Ni}$ with the metalloid upto the limit of solid solution. However, electroless deposition, being a low temperature process, the system is constrained to remain in a single non-equilibrium phase due to its kinetic limitations.

As the metalloid content increases beyond the solubility limit, they produce excessive strain in the crystalline structure resulting in a higher density of defects (Graham et al 1965). Normally one expects that the defects increase with increase in metalloid content and the system becomes microcrystalline. Moreover, when the metalloid content is increased beyond a limit, it becomes difficult to retain the long range order and the system passes into a non-equilibrium amorphous state.

To distinguish between the as-deposited state of an amorphous sample and that of a microcrystalline sample is a difficult task, because responses which are similar to the diffraction studies in the two states. Bagley and Turnbull (1970) provided the first conceptual framework by arguing that on heating, a microcrystalline sample will undergo grain growth to pass on to a crystalline state but the amorphous sample can transform only by nucleation and growth. While there will be a critical temperature only above which it is possible to surmount the activation barrier for nucleation, for the microcrystalline samples the grain growth will take place at all temperatures depending upon the magnitude of the diffusion coefficient.

In the present investigation we have examined the thin films of electroless deposited Ni-P alloys with different P-contents. The thin films were subjected to electron microscopy while heating on the stage of the microscope. The electron micrographs and 
selected area diffraction pattern (SAD) of the films with different $\mathrm{P}$ contents were analyzed to determine the nature of the as-deposited electroless films. Makhsoos et al (1978) undertook similar investigations on electrodeposited films by heating the samples with electron beam itself. However, the extent of heating could not be determined in their investigation. In the present study the sample temperature was monitored throughout.

\section{Experimental}

The Ni-P films were deposited using electroless deposition technique. The details of the bath and conditions are given in table 1 . The concentration of components in the solution was optimized to get a deposition rate of about $800 \AA / \mathrm{min}$. The glass slides, used as substrates, were first washed with liquid detergent (Teepol), then by acid and degreased with hot $\mathrm{NaOH}$ solution. Finally substrates were washed with hot double distilled water. For deposition, these properly cleaned substrates were given a sensitizing treatment (by dipping in a $1 \% \mathrm{SnCl}_{2}$ solution for $60 \mathrm{sec}$ ) and activation treatment (by dipping in a $0.1 \% \mathrm{PdCl}_{2}$ solution for $30 \mathrm{sec}$ ). The film was deposited for about $40 \mathrm{sec}$ for examination under transmission electron microscope.

The samples were chemically analyzed by using wet chemical method, the accuracy of which is less than $\pm 1 \%$. Transmission electron microscopy (Philips model EM $400 \mathrm{~T} / \mathrm{ST}$ with a heating holder PW 6592) was carried out and the general form of the holder was similar to that of other holders for the goniometer stage.

\section{Results and discussion}

Ni-P samples have been deposited by varying the amount of the reducing agent, $\mathrm{NaH}_{2} \mathrm{PO}_{2}$, in the bath so that the composition falls in three ranges--sample I containing $13-15$ at. $\%$ of $\mathrm{P}$, sample II $16-18$ at. $\% \mathrm{P}$ and sample III $19-21$ at. $\% \mathrm{P}$. The composition of the deposits could not be controlled more closely due to limited facilities.

The electron micrograph of sample $I$ is shown in figure 1 . The deposit shows unevenness, but the $\mathrm{SAD}$ as in figure 2 shows a diffuse ring at $2 \theta=1.063^{\circ}$ and $\Delta 2 \theta=0.08^{\circ}$. If it is assumed to be microcrystalline the lattice parameter is approximately $3.46 \AA$ on the average. However, the diffuse ring may have been a characteristic of an amorphous state; while heating this sample the width of the diffuse ring decreases continuously leading to rather sharp rings as given in figure 3 showing

Table 1. Bath and conditions of Ni-P films

\begin{tabular}{lcl}
\hline Plating variables & $\mathrm{g} / \mathrm{l}$ of $\mathrm{H}_{2} \mathrm{O}$ & \\
\hline $\mathrm{NiSO}_{4} \cdot 7 \mathrm{H}_{2} \mathrm{O}$ & 24 & pH-(8.5-9.5) \\
$\left(\mathrm{NH}_{4}\right)_{2} \mathrm{SO}_{4}$ & 50 & adjusted with $\mathrm{NH}_{4} \mathrm{OH}$ \\
$\mathrm{Na}_{3} \mathrm{C}_{6} \mathrm{H}_{5} \mathrm{O}_{7} \cdot 2 \mathrm{H}_{2} \mathrm{O}$ & 88 & temperature-85 $\pm 2^{\circ} \mathrm{C}$ \\
$\mathrm{NaH}_{2} \mathrm{PO}_{2}$ & $(10-45)$ & No stirring \\
\hline
\end{tabular}



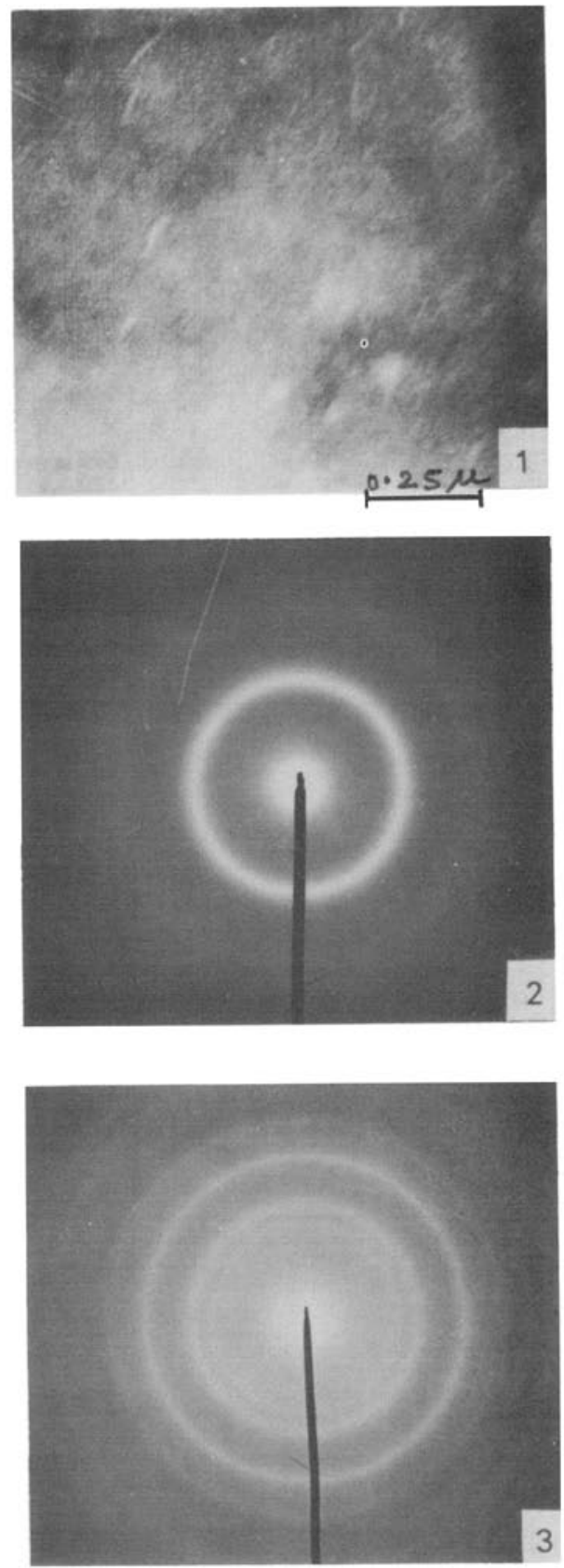

Figures 1-3. Electron micrograph and SAD of Ni-P sample I (13-15 at. \%). 1, 2. As-deposited state. 3. Heated at $350^{\circ} \mathrm{C}$ for $2 \mathrm{~min}$. 

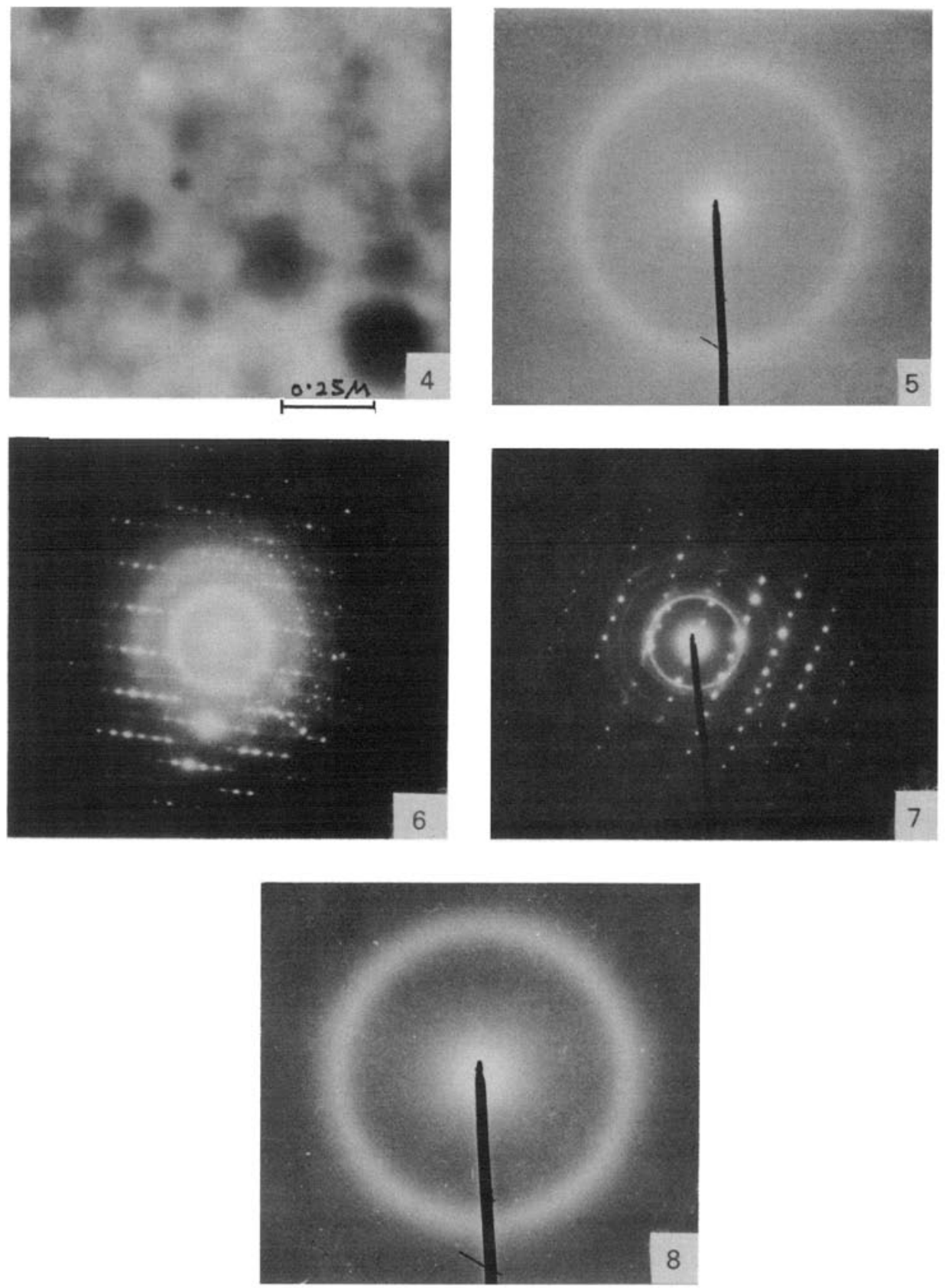

Figures 4-8. Electron micrograph and SAD of Ni-P sample II. 4-7.16-18 at. \%. 4, 5. Asdeposited state. 6 . Heated at $340^{\circ} \mathrm{C}$ for $2 \mathrm{~min}$. 7. Heated at $340^{\circ} \mathrm{C}$ for $2 \mathrm{~min}$ in a region different from that of 6. 8. 19-21 at. \% in as-deposited state. 
the SAD of sample $\mathrm{I}$ heated to $350^{\circ} \mathrm{C}$ for $2 \mathrm{~min}$ in TEM. The reduction in broadening may be attributed to the lowering of defect density and growth of the grains in microcrystalline state. Following the arguments of Bagley and Turnbull (1970), we believe that the sample class $I$ is not in amorphous state but in a microcrystalline state.

The electron micrographs of as-deposited sample II and its SAD pattern are shown in figures 4 and 5. The SAD pattern has a diffuse ring at $2 \theta=1.057^{\circ}$ and its width $\Delta 2 \theta=0 \cdot 13^{\circ}$. Clearly it is identical to that observed for as-deposited sample I excepting for a slight shift in the position of the ring and an increase in width. If the sample is microcrystalline, the higher $\mathbf{P}$ content of the sample will change the lattice parameter causing a shift in position of the ring. The resulting strain may also have been accommodated by larger concentration of defects and reduced grain size. Thus, the diffraction ring has a higher width. But again SAD may be attributed to that of an amorphous state.

On heating sample II to $340^{\circ} \mathrm{C}$ and holding it for 2 min certain areas of the sample showed an SAD pattern as given in figure 6 . The SAD pattern shows broad diffuse rings superimposed over a spot pattern. The retention of width at a high temperature $\left(340^{\circ} \mathrm{C}\right)$ is in sharp contrast with the behaviour observed in sample I. Thus in sample II there is a barrier to the change in state from the one characterized by the broad diffuse ring. In addition, the spot pattern is characteristic of a crystalline state with a crystallite size exceeding the width of focus. It can be inferred that in this region of the sample, an amorphous state transforms to a crystalline state by nucleation and growth. However, in certain other regions of this sample one observes continuous sharpening of broad rings, resulting in sharp rings superimposed on the spot pattern (figure 7). This region appears to consist of microcrystallites and a crystallite of size greater than the size of focus indicating a grain growth and an abnormal grain growth. Sample II contains both amorphous and microcrystalline regions, which may have resulted due to the variation of $\mathbf{P}$ distribution from one region to the other.

Sample III shows an SAD pattern (figure 8 ) and again consists of a broad diffuse ring with $2 \theta=1.055^{\circ}$ and $\Delta 2 \theta=0 \cdot 16^{\circ}$. The position of the ring has slightly been shifted and the width increased obviously due to an increase in P content. However, it is not possible to infer about the structural state of the sample from this SAD pattern. On heating this sample to $345^{\circ} \mathrm{C}$ and holding for $2 \mathrm{~min}$, one observes superposition of broad diffuse rings on the spot pattern similar to that observed in some regions of sample II.

\section{Conclusions}

The electron microscopy study of the Ni-P samples containing 13-21 at. \% P shows that the as-deposited film passes from a microcrystalline state to amorphous state with increasing $\mathrm{P}$ content. At intermediate compositions the microcrystalline and amorphous state co-exist. But it is not clear whether it is a result of composition variation or whether it is possible to achieve these two states together at the same composition. In the composition range of $19-21$ at. \% $\mathrm{P}$ it is amorphous. Several investigations have noted that the samples with low-P are crystalline (Yamasaki et al 1981). Thus, it appears that with increase of $\mathrm{P}$, the as-deposited Ni passes from crystalline to microcrystalline state continuously and then beyond a certain composition to an amorphous state. 


\section{Acknowledgements}

The authors are highly grateful to Dr Kailash Chandra for all facilities at the University Service and Instrumentation Centre and to $\mathrm{Dr} R \mathrm{C}$ Bembi for chemical analysis of the samples. Thanks are also due t - Mr N K Saini for transmission electron microscopy.

\section{References}

Bagley B G and Turnbull D 1970 Acta Metall. 18857

Cortiju R O and Schlesinger M 1983 J. Electrochem. Soc. 1302341

Gorbunova K M, Ivanov M V and Moiseev V P 1973 J. Electrochem. Soc. 120613

Graham A B, Lindsay R W and Read J 1965 J. Electrochem. Soc. 112401

Hedgecock N, Tung P and Schlesinger M 1975 J. Electrochem. Soc. 122866

Maeda H 1970 J. Phys. Soc. Jpn 29311

Makhsoos E V, Thomas E L and Toth L E 1978 Metall. Trans. A9 1449

Pai S T and Marton J P 1972 J. Appl. Phys. 43282

Yamasaki T, Izumi H and Sunada H 1981 Scripta Met. 15177 